\title{
Oral and Maxillofacial Surgery: It's Future as a Specialty
}

\section{Kishore Nayak}

(C) Association of Oral and Maxillofacial Surgeons of India 2012
The specialty of Oral \& Maxillofacial Surgery (OMFS) as we know it now is bound to change drastically in our lifetime. The driving forces for this change are varied and multifactorial, some of which are the cost and length of training combined with the prevailing dichotomy in scope of practice between traditional and expanded areas of practice. The formation of sub-specialties within the specialty-oncologic and reconstructive surgery, facial aesthetics, hospital based practitioners and office based practitioners, dental and medically qualified practitioners complicate the issue further.

At an international level there is a continuous debate about the need to level the playing fields of training in OMFS in different parts of the world and measures implemented towards it. In addition there is an acute need

K. Nayak $(\bowtie)$

International Association of Oral and Maxillofacial Surgeons (IAOMS) 2012-13, Bangalore, India

e-mail: kishorenayak@gmail.com to accept emergence of new technology in practice and training and consider the competition from allied medical and dental specialties.

Internationally the specialty has always been on a pendulous path remaining undecided on the idea of whether it wants to stay a dental specialty or whether it wants to incorporate the medical degree. In the USA where this trend initially started, the specialty is protected by the national, regional and local organizations as a dental specialty without any practical differences in the scope of practice between single or double degree practitioners. In the UK and many parts of Europe, the specialty has taken leaps and bounds towards the medical path of training and it could very well be only a matter of time before the Specialty remains only remotely associated with Dentistry. The primary challenge was a lack of understanding which centered around the debate of whether a medical as well as a dental qualification was required prior to surgical training.

The International Association of Oral and Maxillofacial Surgeons (IAOMS) has debated this vexatious issue ever since its inception 50 years ago. Unfortunately, it took most of the first forty years sparring on this issue within the Association. In 2001, it was finally decided through acceptance of the International Guidelines for Training and Education in Oral and Maxillofacial Surgery, that regional differences in training existed and were acceptable. This document stated that the actual surgical training in the oral and maxillofacial region was what qualified one to become an oral and maxillofacial surgeon in the country or region, regardless of whether one had dental and/or medical qualification.

The issue was very succinctly presented by Laskin [1] where he makes a case for some basic issues to be addressed to resolve the situation. Primarily among these is 
the fact that it is countries and regions that have to decide what standards have to be adopted and fulfilled for one to qualify as a maxillofacial surgeon. A strong case is made for recognizing the adequacy of Dental Education with subsequent Medical training and not necessarily a degree as a prerequisite for completion of qualification in maxillofacial surgery. It is important to recognize that not all people who embrace the specialty have or need to have the same scope of practice.

Laskin attempts to address this problem by dividing the scope of oral and maxillofacial surgery into 3 parts: areas of expertise, competence and familiarity.

Areas of expertise include oral pathology/oral medicine, dentoalveolar surgery, pre prosthetic surgery (including implantology), and maxillofacial traumatology.

Areas of competence involve orthognathic surgery, temporomandibular joint surgery, and local reconstructive surgery.

Areas of familiarity are cleft lip and palate surgery, regional reconstructive surgery, oncologic surgery, craniofacial surgery, and cosmetic surgery.

To be considered an oral and maxillofacial surgeon, one needs to at least include the areas of expertise and competence in his or her scope of practice. Depending on individual training received the criteria that one establishes as their areas of competence or familiarity are interchangeable. However, it is these areas of practice that clearly distinguish the oral and maxillofacial surgeon from the plastic surgeon or the otolaryngologist and sets us apart as a distinct specialty that can offer a unique service to the public.

In spite of all the debates and contemplation it is a moot point whether it will be possible to dissipate fairly uniform training and education across boundaries and borders. The disparity in training standards between different systems worldwide is an identified problem in almost all medical and dental specialties although some refuse to believe that this is indeed a problem. What works well in USA or Europe might not be translatable to the Indian healthcare system or for that matter to any other developing country. However, with pervasive technology aided training such as telemedicine, the gap may be minimized. The transfer of brain power and surgical skills across continents is happening more rapidly and easier than ever before. Leveling this playing field is bound to have an impact on the scope and practice of surgery. Haptic simulated surgery allows surgeons to practice a routine the night before a complicated procedure. Simulated mannequins allow for the trainees to practice cardiac and trauma life support, vena- puncture, tracheostomies, chest tube placements, craniotomies or other high risk procedures without ever jeopardizing a patient.

The IAOMS has recognized the potential power of Information Technology (IT) in changing the face of oral and maxillofacial training world wide and has formed a committee and taken steps which will harness the power of IT in disseminating education world wide. Need based information can be made available to the OMF surgeon at his fingertips and education with updates on the specialty could be continuously received on handheld devices like iPads and smartphones. This would truly make the world of oral and maxillofacial surgery a "flat" one!

The last but very important question that needs to be addressed is one related to economics and income. Are there not many OMF surgeons that have not seen the promised land of delayed gratification? Forced by the economics of the times, coupled with ever increasing pressures of running a successful practice, OMF Surgeon's are narrowing their scopes of work, staying within their offices and clinics, and performing lesser and lesser major procedures. In the battle between the oncologist and implantologist, who will emerge the winner? Can the two coexist within the scope of the same specialty? Are we doomed to be office based procedurists or will we be omnipresent in hospital based practices as well? Do we need extended training for what may distill down to outpatient based practice? There is no doubt at all that the specialty should flourish and coexist these two poles within its scope. For the larger public good and for the sake of the future of the specialty it will be important for these extremes to be symbiotic and move forward. Our future as a specialty will depend not just on our individual economic strengths but also our social and political projections through our professional associations, our ability to meet expectation from the public, who will compare what we have to offer with that of the competition from other surgical specialties. A united front within the diverse yet broad scope of what our specialty offers can make OMFS unbeatable. The future will depend on what we do in the present.

\section{Reference}

1. Laskin M (2008) The past, present, and future of oral and maxillofacial surgery. J Oral Maxillofac Surg 66:1037-1040 